\title{
De la intervención al acompañamiento. Una propuesta para construir conocimiento desde nuestra experiencia encarnada en Trabajo Social.
}

\author{
From intervention to accompaniment. A proposal to build knowledge from our experience \\ embodied in Social Work.
}

Berenice Pérez Ramírez' (1), Laura Lizbeth Osornio Mora² (iD)

\section{Resumen}

A partir de la revisión bibliográfica de los conceptos Acompañamiento Terapéutico (AT) y Acompañamiento Social (AS), así como de un proceso etnográfico realizado en 2019 en el Centro Femenil de Reinserción Social ubicado en la Ciudad de México, nos proponemos discutir la posibilidad de que la noción de intervención desarrollada en Trabajo Social, se desplace hacia el concepto de acompañamiento. Al inicio del proceso etnográfico nos posicionamos desde la implementación de una estrategia de intervención, pero poco a poco emergieron elementos de un proceso de acompañamiento. A partir de ese momento observamos cambios significativos en todas las personas implicadas. Con esto, resignificamos la noción de intervención como un proceso distante, disciplinador y propio de una ortopedia social, para acercarnos a una experiencia encarnada que significa pensar e interactuar con y desde el cuerpo, porque así se genera sentido y se toma posición a partir de la vulnerabilidad y la intimidad donde se producen nuestras relaciones con los otros y con el mundo (Cortés Severino, 2013). Nuestra propuesta es asumir que la intervención nos afecta porque es una forma de establecer un vínculo en el trabajo con otros y aproximarnos al acompañamiento nos previene de sostener visiones correctivas, normalizantes, salvacionistas y paternalistas.

Palabras clave: Trabajo Social, intervención, acompañamiento terapéutico, acompañamiento social.

\begin{abstract}
Based on the bibliographic review of the concepts Therapeutic Accompaniment (TA) and Social Accompaniment (AS), as well as an ethnographic process in 2019, in Women's Center for Social Reintegration located in Mexico City, we propose to discuss the possibility about the notion of intervention developed in Social Work, moves towards the concept of accompaniment. At the beginning of this ethnographic process we positioned ourselves from the implementation of an intervention strategy, but emerge elements of an accompaniment process. From this moment, we observe significant changes in all the people involved. With this, we resignify the notion of intervention as a distant, disciplining process typical of a social orthopedic, to get closer to an embodied experience that it means thinking and interacting with and from the body, because in this way meaning is generated and a position is taken from vulnerability and intimacy where our relationships with others and with the world are produced (Cortés Severino, 2013). Our proposal is to assume that the intervention affects us because it is a way of establishing a bond at work with others and approaching the accompaniment, it prevents us from holding corrective, normalizing, salvationist and paternalistic views.
\end{abstract}

Keywords: Social Work, intervention, therapeutic accompaniment, social accompaniment.

Pérez Ramírez, B. y Osornio Mora, L.L. (2021). De la intervención al acompañamiento. Una propuesta para construir conocimiento desde nuestra experiencia encarnada en Trabajo Social. Itinerarios de Trabajo Social, 1, 7-14. https://doi. org/10.1344/its.voi1.32484

\section{Introducción.}

Proponemos mirar la intervención como una operación analítica porque implica articular saberes epistemológicos, teóricos y metodológicos antes, durante y después del encuentro con otro sujeto, sea individual o colectivo, para crear estrategias con miras a un diálogo inter y multidisciplinario. En este sentido, las posibilidades de intervención están directamente relacionadas con la apertura crítica epistémica, su huella metodológica y nuestra biografía, porque a partir de ésta, nos decantamos hacia ciertas direcciones que adquieren un sentido y significado peculiar para nosotras/os.
A partir de la revisión bibliográfica de los conceptos Acompañamiento Terapéutico (AT) y Acompañamiento Social (AS), así como de un trabajo de campo en el Centro Femenil de Reinserción Social en 2019, nuestro objetivo es discutir la posibilidad de que la noción de intervención desarrollada en Trabajo Social se deje influenciar por el concepto de acompañamiento y nos preguntamos: ¿qué podemos dialogar con los conceptos de acompañamiento terapéutico y acompañamiento social?, ¿el acompañamiento le brinda horizontalidad a lo que hemos concebido como intervención?

Nuestra apuesta es que, al considerar elementos del
ACEPTADO: 27/11/2020 PUBLICADO: 21/01/2021

2 Escuela Nacional de Trabajo Social, UNAM.

$\square$ Berenice Pérez Ramírez. Escuela Nacional de Trabajo Social (UNAM). Avenida Universidad, 3000, C.U. Coyocán, C.P. 04350. Ciudad de México, CDMX. bereramirez@comunidad. unam. $m x$ 
acompañamiento en la intervención social, proporcionara elementos que nos permitan desarrollar procesos cercanos y apostar por un trabajo más horizontal, con efectos duraderos en ambas direcciones: para las personas con quienes nos encontramos y para quienes en prendemos los procesos de incenención. El objetivo de AT y el AS en las estrategias de intervención de Trabajo Social podemos desplazar del concepto intervención las nociones de que es un proceso distante, disciplinado normalizador y moralizante, para acercarnos a una periencia encarnada que significa pensar e interactua con y desde el cuerpo, porque así se genera sentido y se toma posición a partir de la vulnerabilidad y la intimidad donde se producen nuestras relaciones
con el mundo (Cortés Severino, 2013).

con el mundo (Cortés Severino, 2013).
A partir un trabajo de campo situado, apuntamos que pone una forma de conocimiento desde la experienta manifiesta en el cuerpo, y principalmente nos permitió descentrar la idea de que la construcción de conocimie to solo es a través de la cognición y la memoria. El concepto experiencia encarnada deviene de la articulación del concepto memoria incorporada (Fassin, 2016) y el de individualidad encarnada (Kontos, 2005), que indica que el cuerpo no es una tabula rasa, ahí se libran procesos reflexivos, tiene una habilidad inherente para aprender vehicular sign

El presente escrito se divide en cuatro apartados. En primer apartado presentamos una síntesis de cómo estamos concibiendo la intervención. En el segundo apatado desarrollamos las ideas centrales sobre Acompañ miento Terapéutico y el Acompañamiento Social. En tercer apartado detallaremos la ruta metodológica que seguimos en el acompañamiento con mujeres que está ubicadas en un área psiquiátrica dentro de una cárcel co la Ciudad de México. En el cuarto apartado presentamos tado hacenos una discusión y ańlisis de resultados.

2. Breviario sobre la intervención de Trabajo Social.

La intervención en Trabajo Social en el contexto mexica no y latinoamericano ha sido comúnmente denominad intervención social o in tervención en lo social. Entre los principios básicos de este concepto encontramos que opera en un registro distinto a la investigación, recae con y sobre los sujetos concretos en campo y sropone enTello Peón, 2010). Por el contrario, proponemos mirar intervención como una operación analítica porque imp ca articular saberes epistemológicos, teóricos y metodo sujeto, sea individual o colectivo, para crear estrategias con miras a un diálogo inter y multidisciplinario. La intervención no es sinonimo de acciones o activida des, es decir, la intervención implica hacernos preguntas como las siguientes: ¿Con quièn(es) intervenimos? ¿Para qué lo hacemos? :Cual es mil lugar en la intervención situación en la que intervenimos? ¿Los sujetos, es decir, nuestras/os interlocutoras/es, que opinan de esta interVión? y ¿Que me ocurre a mi durante este proceso? testas preguntas siempre estarán alrededor de las estra

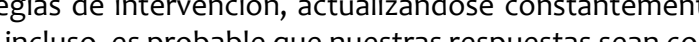
trictoras yrobable que nuestras respuestas sean confique de manera importante conforme avanose modprue de manera inportante co

Existe una línea, basada en el pensamiento de Foucault como una ortopedia social (Míguez Passada, 2017), con laras tendencias hacia el disciplinamiento y normalización de ciertos sectores subalternos. Con ello, apuntan al origen contradictorio del Trabajo Social como profesión, en tanto tuvo el objetivo de redireccionar y corregir a los cectores manentes de esta noción alrededor de las intervenciones, también es cierto que se han ramificado distintas perspectivas sobre lo que se entiende por este dispositivo (Carballeda, 2010).

a noción de intervención es una "construcción socio histórica, que actúa en la compleja trama social" (Muñoz, 2011, p. 87). Los procesos de intervención se sostienen epistemologias concretas y en ocasiones en diversos (y contradictorios) supuestos epistemológicos. La parte pero siva es lo más visible de nuestras intervenciones, Es decir, para apuntar a una intervención y no sólo un. serie de acciones inconexas debemos explicitar los conceptos, las nociones y el posicionamiento político de la propuesta. En caso contrario, la intervención será sólo un gesto vacío, inmediatista e inconcluso. Por tanto, la intervención "no se define por el objeto o la unidad de

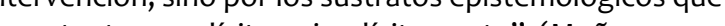
sustentan explicita o implicitamente" (Munoz, 2011, p. 93). El sustento epistemológico en la intervención no poce ser visto únicamente como requisito, sino como un mamente ligada a nuestros preceptos sobre el mundo y a vida social.

En este sentido, las posibilidades de intervención están directamente relacionadas con la apertura crítica epistémica y su huella metodológica, de cómo construimos un problema, como entablamos el diálogo con nuestros interlocutores para plantearnos las preguntas que enyuzaremos, cuales son los supuestos que consideramos cómo se articulan para que concibamos que algo addure el estatus de problematcica. En este proceso, no hridad nos decantamos hacia ciertas direcciones que adquieren un sentido y significado peculiar para nosotras/ nn Trabajo Social estamos en constante contacto con lases y grupos subalternos (Crehan, 2016), es decir, "quellos que han sido "tutelados" o catalogados como "quienes no tienen voz", que viven en condiciones de desigualdad o que no pueden controlar los modos en los que se les enuncia, porque en las condiciones de oprepelar a quienes los gobiernan La intervención social se despliega en contextos marcados por una creciente dife- renciación social, pobreza y explotación. En este sentido, el y la profesional que intervienen, además de constatar estos niveles crecientes de complejidad, deben atender a los marcos institucionales en los que se desenvuelven. Por tanto, lo social debe entenderse como un proces total que debe ser enmarcado en contexto histórico y do por la explotación y el consecuente auge de las clases subalternas. Siendo así, la intervención se nos presenta como compleja y contradictoria. Por ello, la intervención no puede emanar de buenas intenciones porque el ca pitalismo puede reformarse, pero no por caridad, "sino por la capacidad organizativa de los trabajadores y las clases subalternas" (Macip, 2008, p. 31).

En este marco, asumimos que la intervención nos afecta porque es una forma de establecer un vínculo en trabajo con otros y surge "una trama de afectos", de 2002 . p. 13). Ello significa que no estamos por fuera de intervención, el trabajo con el otro nos implica y pued transformar nuestras posiciones en un proceso inacab do. Debemos estar alerta de visiones correctivas, norma lizantes, salvacionistas y paternalistas que prevalecen en algunos espacios formativos y profesionales. Por ello, nos preguntamos iqué podemos dialogar con los conceptos de AT y AS?

3. ¿Qué son el acompañamiento terapéutico y el acompañamiento social?

Por un lado, la figura de Acompañante Terapéutico se origino a partir de otras formas enfocadas a acompaña (Rossi, 2007). Algunos ejemplos fueron los acompaña tes llamados "monitor de tiempo libre" en España o "anmateur" en Francia. En Canadá, se introduio la figura de "interviniente clínico" para acompañar los tratamientos de personas con psicosis, mientras que en Inglaterra y Belgica se crearon los "dispositivos de enlace". Por otra parte, en Argentina y Brasil se llamo "amigo cualificado" y después tomaría el nombre de acompanante terapéut co (Saiz Caldósy Chevéz Mandelstein, 2009).

(Storapéutico provienen de Espana, surgiendo en el marco de los gentina, varios autores (Pulice y Rossi, 1994: Nivoloni, 2008) coinciden que es el país donde ha tenido mayo auge y desarrollo. El AT surge aproximadamente en década de los años 70 y está articulado con los movimientos que se produjeron en torno a los cuestionamientos sobre los métodos usados en esa época para tratarla salud mental, generando las condiciones para imple

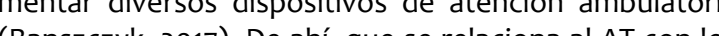
(Banszczyk, 2017). De ahi, que se relaciona al AT con los la desmanicomialización 4 (Serra Hurtado, 2003) promo viendo la salida de la persona del ámbito clínico y tratarle en su contexto cotidiano y social.

EIAT es un dispositivo, es decir, una red, que posee una singularidad, es de baja exigencia ya que el acompañamiento requiere de cierta flexibilidad a lo largo de las etapas que le conforman, para adaptamos a las necesidades y características de cada persona; no es directivo porque busca que la persona decida o descubra lo que necesita. Tiene una perspectiva Clinica y socio-comunitaria ya que abarca eambito linicoinstitucional pero tambien busca in-

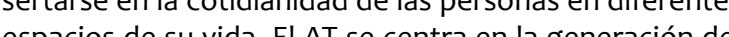
vínculo a suvia. El AT se centra en la generación de social (Serra Hurtado, 2003; Chévez y Montuori, 2017;

establecen que las bases del AT surgen en el psicoanálisis (Macias, 2013; Martiarena et al., 2017), sin embargo, varios autores se posicionan dentro otros enfoques como el psicodrama (Moreno, 1993; Che vez y Montuori, 2017) y la psicología social (Costa, 2017; (202) 20 . Autores como Kuras de Mauer y Res. (2004), Saiz Galdós y Chevéz Mandelstein (2009) de un trabajo interdisciplinario. Entre las principales funun espacio para pensar, orientar en el espacio social, ampliar una mirada del mundo, contener a nuestro/a in terlocutor/a, permitirle que se organice psíquicamente, registrar y ayudar a desplegar la capacidad creativa de persona e intervenir en la trama familiar (Kuras de Maue y Resnizky, 2004).

Por otro lado, el AS es una modalidad con mayor concep tualización y práctica en Trabajo Social y comprende una en un mayor grado de vulnerabilidad con el fin de que se incorporen a la sociedad (Alonso y Funes, 2009). García Muñoz (2012) establece que:

El termino AS empieza a ser utilizado por los profesionales de Trabajo Social y la educación en torno que luchaban en contra de la exclusión social y a favor de la integración de niños con necesidades educativas especiales de los países de habla francesa. (p. 41)

El AS se considera un método de intervención profesional temporal, de intensidad variable, basada en el derecho a la ciudadanía de una atención social personalizada. El objetivo de este acompañamiento es desarrollar potencialidades y capacidades de la persona hacia el prota gonismo de su propio proyecto de vida (Red Navarra El AS versa principalmente en tres dimensiones:

Gráfico 1. Dimensiones del Acompañamiento Social.

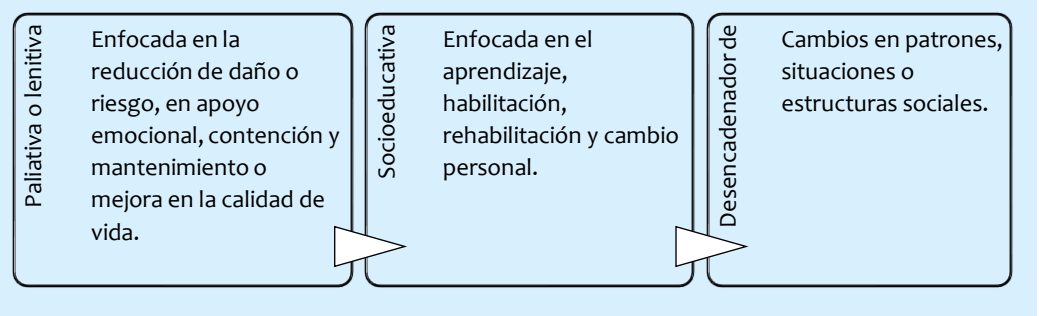

El AS puede darse en diversos ámbitos, en la salud, lo educativo, Io juridico, lo comunitario, lo familiar, etc., co diferentes sectores de la población, sean niños, adultos mayores, personas privadas de la libertad, mujeres que
viven violencias, etc. (Borja, 2013): 
Tiene un claro horizonte de autonomía y liberación de la persona, no parte de un esquema predeterminado, con etapas definidas, sino que se adecua al momento circunstancias vitales. En ese sentido, podemos hablar de una ausencia de proceso. (p. 128)

El acompañamiento social no solo puede ser person lizado, sino que también se puede aperturar en grupos contrar:
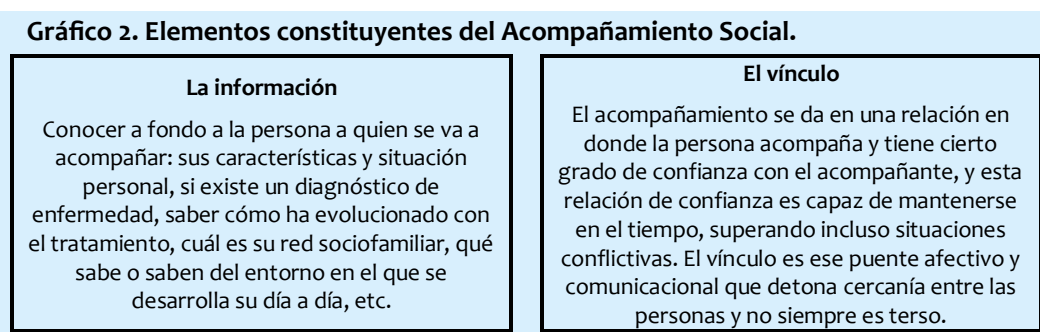

personas yne semempre es esterso

La flexibilidad

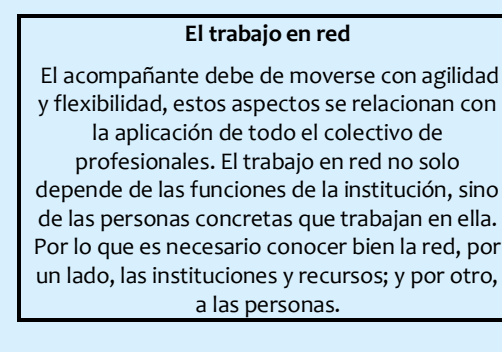

intervención. La flexibiliidad necesita un

escucha, conocery ydaptarse a las neceses

a: Elaboración propia a partir de Boria (201)

A lo largo de este recorrido hemos plasmado algunas de acompañamiento. En la siguiente tabla colocamos las diferencias y similitudes que identificamos:

Tabla 1. Diferencias y similitudes entre el Acompañamiento Terapéutico y el Acompañamiento Social

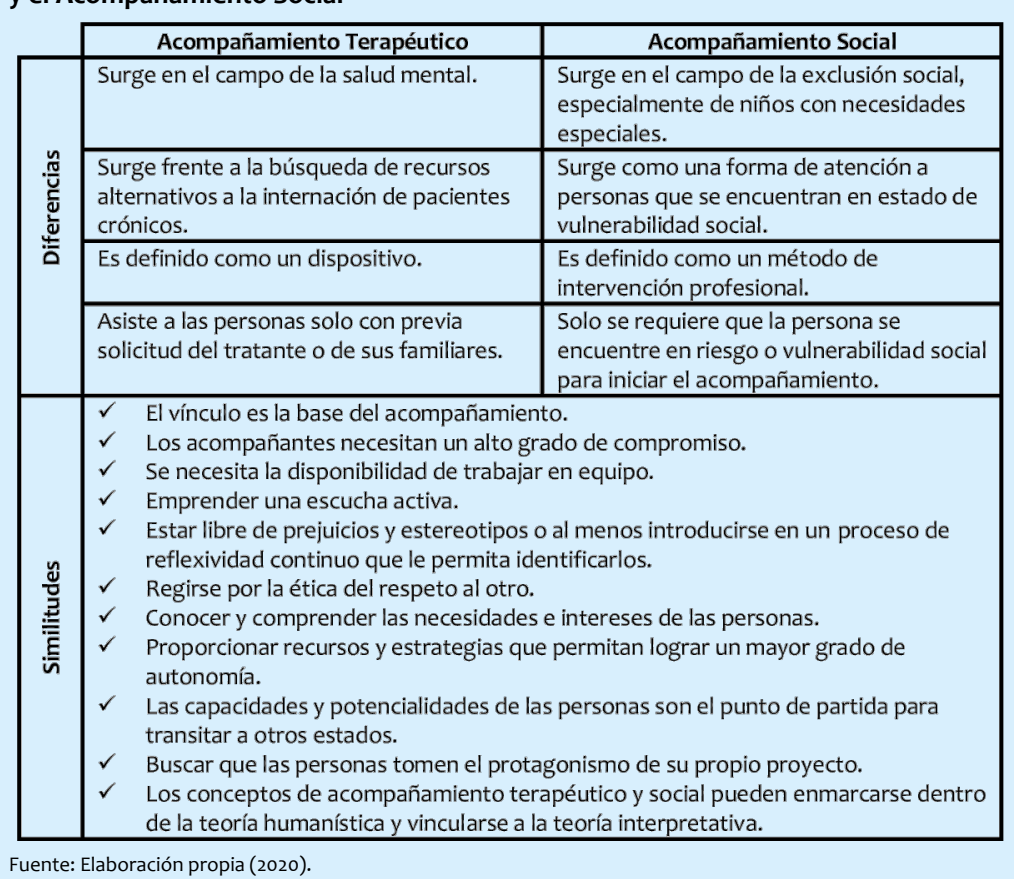

El AT y el AS se encuentran diferenciados por varios aspectos y a su vez conectados por similitudes. Queremos
resaltar cuatro de ellas: por un lado, ambas son herra- mientas de intervención que pueden proporcionar nue vas perspectivas, por ejemplo, sobre como nos posicionamos los y las trabajadoras sociales en los procesos de der necesidades, demandas y nuevas rutas para atenpersonas que lo requieran, al ritmo que as lo desellas y en una posición menos jerárquica. Por otro desee acompañamiento solo se puede lograr con base en el vínculo a construir con los y las interlocutoras, por lo que es esencial asumir que éste se da y apostar por la reflexividad como método permanente para indagar en torno a nuestros vínculos. Por último, los conceptos de acompañamiento terapeutico y social se enmarcan dentro de la teoria humanistica y la teoría interpretativa, colocando en el centro al ser humano, considerando que (Ander-Egg, 2011).

4. Ruta metodológica.

El objetivo de este artículo es mostrar que si se retoman ción de Trabajo Social, podemos desplazar del convenintervención las nociones de que es un proceso distante disciplinador, normalizador y moralizante, para acercarnos a una experiencia encarnada. A partir del trabajo de campo apuntamos que el acompañamiento como experiencia encarnada supone una forma de conocimiento desde la experiencia manifiesta en el cuerpo, y principalmente nos permitió descentrar la idea de que la construcción de conocimiento solo es a través de la cognición y la memoria, pues las formas de conocimiento no solo son textuales, sino sensoriales y sociales (Aguilar Díaz, 2014; Esteban, 2004).

colizó conjuntamente ra "práctica de especialización". Esta asignatura la cursan en el último año de la licenciatura en Trabajo Social en la Escuela Nacional de Trabajo Social de la Universidad Nacional Autónoma de México. La metodología desarrollada se acercó a los supuestos de la lnvestigación Acción Participativa (Ortega et al., 2019; Urdapilleta Carrasco y Limón Aguirre, 2018; Paredes-Chi y Castillo-Burguete, 2018), ya que nos interesamos porque las mujeres tuviedesarrollar fueran propuestas por ellas. Nuestra premisa fue tratarlas como sujetos que saben de sí, de su condición psicosocial y del encierro, no pretendíamos "darles voz". En ese sentido, nos ubicamos como agentes externas que queríamos entrar en diálogo con ellas y accionar en la medida de lo posible una horizontalidad, ésta se manifesto a través de nuestra participación con ellas en las actividades y no pedirles algo que no podíamos dar. al día (6 sesiones) para conocernos y saber qué 4 horas des les interesaban y posteriormente proponerlas entre agosto y noviembre del mismo año. En este segundo periodo asistimos dos veces por semana 4 horas al día (27 sesiones).

Esta estrategia surgió de un análisis contrapunteado entre lecturas de diversos temas y lo que emergio en nuesquienes nos dijeron quiénes eran, qué les gustaba, qué extrañaban, qué anhelaban, etc.

Dividimos la estrategia de intervención en cuatro fases. La primera fase fue de conocimiento mutuo, actividades sencillas para compartir y generar nuestros primeros diálogos. La segunda parte, consistió en desarrollar actividades más organizadas, habla un/a coordinador/a del grupo de práctica, se establecía un objetivo para cada se generaban preguntas de discusión para repensar en
tre todas. Al inicio de esta segunda fase, todavía no se conformaba un grupo, en este momento establecimos que para tomar la palabra debían levantar la mano, no tenían que interrumpir a quien hablaba, ni burlarse de que otra compañera decía, esto lo hicimos porque solían discutir y se burlaban de las mujeres con mayor desventaja, por ejemplo, quien deliraba, quienes eran mayores o se las etiquetaba con "fuertes problemas mentales" infancia, violencias, abuso sexual, discapacidad, locura, encierro, miedos, culpa, redes de apoyo, entre los más importantes; en esta fase se conformó el grupo, en cuanto llegábamos nos saludábamos siempre con un abrazo ellas tomaban su silla y se sentaban en círculo, las participaciones eran continuas, disminuyeron las agresiones entre ellas y comenzaron a hablar de sí mismas, nos lanzaban preguntas importantes: ique violencias han v. vido? ¿por qué estudian Trabajo Social? ¿qué sienten en la coordinación de algunas sesiones. La cuarta fase, dispuso como una fase de próxima despedida, reitera mos continuamente que nos retiraríamos, debido a que el proceso de esa práctica terminaría y nos enfocamos a proponer espacios colectivos entre ellas, "no nos ne cesitan porque se tienen a ustedes" fue nuestra consig. na. Observamos que comenzaron a dialogar y a convivi más, a compartise desde un chocolate hasta un pan, expusieron sus desacuerdos y peleaban menos.

Las técnicas utilizadas fueron la observación directa $d u-$ ción Social (CEFERESO), la obro Federal de Readaptalos talleres, conversaciones clave antes o después de los talleres y un manejo minucioso del diario de campo porque no se nos permitió ingresar grabadora de voz, as que nos guiamos por las notas de campo que fueron de talladas y descritas inmediatamente después de salir de centro. Las notas de campo incluyeron descripciones de espacio donde se desarrollaban las actividades, los obje tos y personas que estaban presentes, los diálogos y las con nosotras, así como las emociones expresadas.

\section{Resultados.}

El encierro enferma y los efectos del encierro impactan las principales problemáticas que observamos en las mu jeres del área psiquiátrica fueron: no pueden participar en las actividades generales del centro, especialmente en el centro escolar, viven un doble encierro que detona conflictos y problemas entre ellas, se enojan con fa cilidad, se molestan unas a otras, se aíslan, agreden quienes consideran más vulnerables y se autolesionan. En este espacio se observan aún más restricciones que en población general, ello se puede identificar a través de la infantilización, la amonestación, la observación m y controlar a las mujeres. La infantilización consiste tratarlas como "niñas que no saben"; ante ello, la amojeres de estos dormitorios están expuetas a. Las muvación minuciosa por parte de las técnicas penitenciarias y la custodia, no pueden escapar de la mirada regulatoria. Todo ello incrementa la hostilidad entre ellas y con el paso del tiempo, observamos que el doble encierro y hechos específicos las tienen en un estado de tensión latente que muchas veces se transforma en agresión protesta.

La propuesta de intervención se fue situando cada vez más como un acompañamiento, porque evitamos exig es común en las actividades penitenciarias), la asistencia variaba de acuerdo al estado de ánimo de las mujeres, así que fuimos flexibles y reivindicamos que las puertas del taller siempre estaban abiertas para cuando ellas quisieran asistir, buscamos adaptarnos a sus necesidades a las características de cada persona. Asumimos asistir en su vida cotidiana y principalmente apostamos por generación del vínculo entre nosotras y con ellas. No fue posible establecer un vínculo con sus familias pues la mayoría ha perdido contacto, además no reciben visitas y la colectiva sobre distintos temas y asuntos se volvió el nú cleo de nuestra estrategia.

6. Discusión: transitar de la intervención a acompañamiento.

La primera lección aprendida fue que el campo nos intere. Por un lado, la propuesta que formulamos como les eran nuestros postulados un epistemolóógicos y metodo lógicos, así como nuestra apuesta personal y política. Las actividades tenian el objetivo de que nos conociéramos porque sabiamos que primero necesitábamos escuchar las y que ellas nos conocieran, que nos platicaran quieo saber el delito que habían cometido no era suficiente) y preguntarles qué les interesaba.

Nos negamos a pensar que la intervención se dirigiera al rescate o la reinserción de las mujeres, así también, nos distanciamos del objetivo de la institución, lo cono cíamos pero no nos adheríamos a él. Como en toda investigación/intervención, fuimos construyendo nuestro problema a partir del encuentro con el campo, de una red de relaciones que permitieron la comprensión de tra mas más amplias. Nuestro compromiso politico se manque rechaza la separación entre lo político y lo personal entre pensamiento y emoción y recuperar, en todo momento del proceso de investigación/intervención, las experiencias de las mujeres y nuestras experiencias.
Esto repercutió también en las técnicas. Aquello que llamamos observación participante por momentos se transformó en participación observante porque tambien conpartíamos ideas, discutíamos temas que ellas proponía 
y nos sentíamos interpeladas con situaciones que nos compartían. En ese sentido tanto como en los procesos de investigación, en la intervención "[La trabajadora so-
ciall] es también sujeto de la investigación ya que se inciall] es también sujeto de la investigación ya que se in-
volucra conjuntamente con los demás, en el proceso de investigación, aprendizaje y acción, lo que implica dejar de lado su neutralidad" (Delgado Ballesteros, 2010, nían un lugar de enunciación propio y en la relación que construimos con ellas también encontramos el nuestro para entrar en diálogo.

Las formas de hacer intervención empezaron a vers trastocadas por la noción de acompañamiento. Por ejemplo, en un inicio pensamos solicitarles un consentmiento informado, no obstante, al llegar a este espacio nos dimos cuenta que algunas de ellas no sabian leer escribir y encontramos a una mujer con demencia. En un consentimiento informado? Por ello, ampliamos noción de cuidado del otro que, por ejemplo, no se re solvía en hacerlas firmar este documento, de fondo, importante es que no nos proponíamos "extraer" infor mación sino acompañar, escuchar, dar soporte, sentirnos también escuchadas por ellas y hacerles saber que estabamos ahi mientras se transmitian experiencias, narraban momentos o cuando guardábamos silencio. La atención al cuerpo, a los cuerpos, fue fundamenta te e implícitamente, se genera una interconexín entre la experiencia corporal propia y con las/los interlocutores en la investigación/intervención. A lo largo de las experiencias en espacios de encierro, hemos corroborado como el cuerpo "se cansa" en cada ingreso, enferm mos cuando las sesiones exigen contención emocional o cuandolas experiencias narradas "nos rebasan" y no podemos digerirlas. Quizá resulte obvio que la observación trae consigo la implicación de quien investiga con sus interlocutores, pero nuestra inmersión conllevó la implmás nos solicitaban las mujeres y que nosotras tama vé buscamos: abrazarnos, estar cerca, sentirnosy mirarnos. Ahmed (2015) indica que el objetivo de subordinar tas emociones en un proceso funciona también para subordinar lo femenino y el cuerpo. Frente a ello nos propus mos asumir que en los encuentros circulaban emociones ligadas a nuestras corporalidades. De esta manera, las emociones no son del orden biológico ni individual sino prácticas relacionales, por tanto, culturales y sociales. Emociones comoldo enserion

Frente a ello, Ahmed (2015) propone que debemos los vínculos al ámbito de la acción política. En nuestro ejercicio, el dolor fue una emoción recurrente, así que traerlo a la acción política significó, por un lado, reconocer que el dano tiene una historia y, por otro, icentificar las condiciones de posibilidad para que estas historias sean escuchadas de manera justa: "una escucha imposible de este tipo solo es posible si respondemos a un do2015. p . 71) sevuelve necesario un trabajo de traducción,
Por tanto, no se trata de sentir o empatizar con el dolor

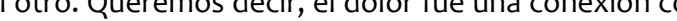
llas, pero solo aprendimos a leer el dolor en la otra y reconocer que no hay forma de saber de el con exactitud, sino solo asumir que estamos conectadas. En cad una de las sesiones se mostraron y se narraron efectos nosotras) al grado de dejarlas sin referentes y por eso el suicidio es tan común en este espacio.

Al final de nuestras sesiones, había un grupo de 15 muje res que asistían con regularidad. A nuestra llegada saldábamos a todas con un beso y un abrazo. Nos coloca bamos en círculo e iniciábamos la reflexión colectiva. El acompañamiento en cada sesión lo viviamos como una experiencia encarnada, porque cuando el grupo de prác cárcel, reunia para hacer un balance de las sesiones en gría cuando identificábamos grupalmente una mejora o un cambio en las relaciones entre las mujeres y angustia cuando se acercaba la despedida. La construcción de ese conocimiento se manifestó en nuestros cuerpos o, en las palabras de Ahmed (2015), el conocimiento está ligado a lo que nos hace sudar, estremecernos, temblar, etc.

\section{Consideraciones finales.}

hemos concemiento le brinda horizontalidad a lo que como intervención. A partir del traba

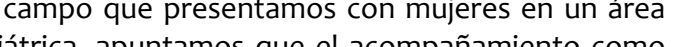
psiquátrica, apuntamos que el acompanamiento como experiencia encarnada supone una forma de conoci-
miento desde la experiencia que se marca en el cuerpo. La estrategia de intervención retomó las características compañamiento terapéutico y social, entre ellas: tro vínculo con las mujeres se fortaleció a través se manvivencia; nuestro compromiso ético y pollico decir, posicionar una escucha justa; el trabajo en equipo por parte del grupo de práctica se consolidó, si bien existieron desacuerdos, estos nunca fracturaron nuestra relación y, al contrario, consolidaron nuestros lazos; conforme conociamos cada vez más a las mujeres, los posibles prejuicios que teníamos del espacio y de ellas se sí, de su condición psicosocial y del encierro; nos acen de mos en la medida de lo posible a comprender sus planteamientos e intereses; las mujeres adquirieron cada vez más el protagonismo del proceso y la reflexión colectiva sobre distintos temas y asuntos se volvió el núcleo de nuestra estrategia.

\section{Bibliografía}

Aguilar Díaz, M. A. (2014). Corporalidad, espacio y ciudad: Tutas conceptuales. En García Andrade, A. y Sabido Ramos, O. (Coords.), Cuerpo y afectividad en la so-

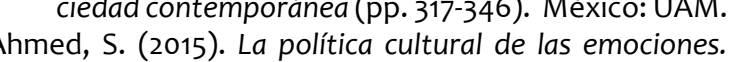
México: PUEG, UNAM

Alonso, I. y Funes, J. (2009). El acompañamiento social 42 (maco-asosto) 28-46. Recupertencion se htp:/lhdil. handle.net/11162/28810
Ander-Egg, E. (2011). Humanismo y Trabajo Social. Inte racción y perspectiva: Revista de Trabajo Social, (2), 142-151. Recuperado de https://es.scribd.com/ document/12629
bajo-Social-Cap-3

Banszczyk, B. (2017). Representación social y rol del acompañante terapéutico que poseen profesionales de saticos (Tesis de licenciatura). Universidad Siglo 21 . Recuperado de https://repositorio.uesigloz21.edu. ar/bitstream/handle/ues21/13768/BANSZCZYK\%20 BRIAN.pdf?sequence=18isAllowed $=y$

Barranco Expósito, C. (2004). La intervención en trabajo social desde la calidad integrada. Recuperado de http://rua.ua.es/dspace/handle/10045/5592

Borja, A. (2013). El acompanamian to social personalizado como formula innovadora de respuesta a personas Servicios Sociales, 54, 127-37. Recuperado de https: dialnet unirioia.es/servlet/articulo?codigo=4536463

Carballeda, Alfredo J.M. (2010). La intervención en lo social como dispositivo. Una mirada desde los escena rios actuales. Revista Trabajo Social UNAM, Época VI, 1 (diciembre), 46 - 59. Recuperado de http://www. revistas.unam $\mathrm{l} / 23881 / 22460$

Castillo Parada, T. (2018). Subjetividad y autonomía: significados y narrativas sobre la discontinuación de 529. http psiquirgicos. Salud colectiva, 14 (3), 5

relaciones que trasforman. España: Equipo del observatorio del tercer sector de Bizkaia.

Cea Madrid, J. C. y Castillo Parada, T. (2018). Locura Neoliberalismo. El lugar de la antipsiquiatría en salud mental contemporanea. Política y sociedad. 55 (2), 559-574. https://doi.org/10.5209/POSO.5727 Chévez, A. y Montuori, A. (2016). Psicodrama y teatro
espontáneo como herramienta conceptual para acompañamiento terapéutico de personas con en fermedad mental grave. En M.L. Frank, M. Costa y D. Hernández (Comps.), Acompañamiento Terapéu tico. Clínica en las fronteras (pp.55-66). Córdoba: Brujas.

Consejo Nacional para Prevenir la Discriminación. (2007) Convención sobre los derechos de las personas con discapacidad. Recuperado de http://www.cona pred.org.mx/documentos_cedoc//Convencion\%2 nas\%20con\%20Discapacidad-Ax.pdf

Constant, C. (2011). Relaciones entre internas y guardias en el penal de muieres de Lima: Análisis de desigutdades de trato entre las internas y las guardias en el penal de mujeres de Lima. Bulletin de l'Institut Français d'Études Andines, 40 (2), 411-418. http://do org/10.400o/bifea.1550

Cortés Severino, C. (2013). El diario como práctica narrativa y visual. En Castillo Ballén, S. (Ed.), Investiga ciones sobre el cuerpo. Relatorias del encuentro "

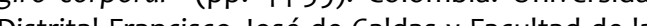
Artes-ASAB.
Costa, M. (2017). Caminos/Formación del at y políticas publicas: El acompañamiento terapéutico en proceso de trasformación en las practicas de salud mental. En Chévez, A. (et al.): compilado por Frank, M.L., Costa, M. y Hernández, A. D. Acompañamiento terapéutico: Clinica en las fronteras (pp. 239-248). Córdoba: Brujas.

han, K. (2016). Gramsci's Common Sense: Inequality

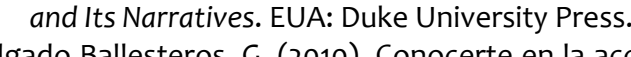

el intercambio. La investigación acción participativa. En Blazquez Graf, N., Flores Palacios, F. y Ríos Everardo, M. (Coords.). Investigación Feminista: Epistemología, Metodología y Representaciones So ciales (pp. 197-216). México: UNAM.

ección General del Instituto de Reinserción Social. (2018). Estrategias de Reinserción Social. Propuestas cura gob mx/storage/app/uploads/public/5b6/b5b/019/ 5b6b5bo19cocf579067633.pdf

Dosil Mancilla, F. (2019). La locura como acción política. El movimiento antipsiquiátrico en México. Revista Electrónica de Psicología Iztacala, 22 (1), 628-645. Recuperado de https://www.medigraphic.com/pdfs/ epsicologia/epi-2019//epi191zf.pdf

po. G.L. (2004). Antropología del cuerpo. Cénero, itinerarios corporales, identidad Fassin, D. (2016). La fuerza del orden. Una etnografía del accionar policial en las periferias urbanas. Argentina Siglo XXI editores.

Galende, E. (2015). El conocimiento y prácticas de salud mental. Argentina: Lugar Editorial. cía Muñoz, M. (2012). Adolescentes en conflicto con la ley. Una terminologia desde lo social y el acompa namiento. Ts nova: trabajo social y servicios sociales, 5 (10), 37-54. Recuperado de http://roderic.uv.es handle/10550/33455

social en la sociedad conter

comello, C. (2013). Género, Drogas y Prisión. Experiencia de mujeres privadas

uber, R. (2001). La etnografía. Método, campo y reflexivi dad. Argentina: Grupo Editorial Norma.

M. (2009). Vigilar y castigar. Nacimiento de la prisión. México: Siglo XXI Editores.

cer lugar. En ML. Frank, M. Costa y D. Hen AT: $H$ (Comps.), Acompañamiento Terapéutico. Clínica las fronteras (pp. 83-87). Córdoba: Brujas.

ernández González, M., Vispe Astola, A. y Gracia-Valdecasas Campelo, J. (2019). De brujas, putas y locas narrativas de género y su influencia en el diagnóst co. Norte de Salud Mental, XVI (60), 33-41. Recupe-
rado de https://dialnet.unirioja.es/servlet/articulo?rado de https://d
codigo=7099338

Tia. (2005). Embodied selfhood in Alzheimer's

Rethinking person-centred care. Dementia, 4 (4) 553-570. 
Kuras de Mauer, S. y Resnizky, S. (2004). Acompañantes terapéuticos: Actualización teórica-clínica. Argentina: Letra Viva.

Macías Terríquez, M. A. (2013). El Acompañamiento Terapéutico con orientación psicoanalítica. Elementos principales y la narrativa de un ejercicio de su narrativa de su aplicación. Revista electrónica de Psicología Iztacala, 16 (4), 1214-1236. Recuperado de https://www.medigraphic.com/cgi-bin/new/resumen. cgi?IDARTICULO=69631

Macip, R. (2008). Clase y discriminación. Revista Ciencia, (abril - junio), 23 - 31. Recuperado de https://www. revistaciencia.amc.edu.mx/images/revista/59_2/ PDF/05-614-p23-31.pdf

Martiarena, N., Dragotto, P. A., Degiorgi, G., Godoy, J. C., Brussino, S., Scherman, P., Muñoz G. C., Marino, J., Peiró, J., Salvador, A., Arcanio, M., Aybar, A. y Falavigna, C. (2017). Manual curso de nivelación: Tecnicatura Universitaria en Acompañamiento Terapéutico. Argentina: Asociación Cooperadora de la Facultad de Ciencias Económicas de la U.N.C.

Mier, R. (2002). El acto antropológico: la intervención como extrañeza. Revista Tramas, 18 y 19 (julio-diciembre), 13 - 50. Recuperado de https://publicaciones.xoc.uam.mx/MuestraPDF.php

Míguez Passada, M.N. (2017). Genealogía, poder y disciplinamiento en el trabajo social: un análisis desde Foucault. Fronteras, 10, 163 - 174. Recuperado de https://www.colibri.udelar.edu. uy/jspui/bitstream/20.500.12008/10197/1/RF_Miguez_2017n10.pdf

Muñoz, G. (2011). Contrapuntos epistemológicos para intervenir lo social: ¿cómo impulsar un diálogo interdisciplinar? Cinta moebio, 40, 84 - 104. Recuperado de http://www.facso.uchile.cl/publicaciones/ moebio/40/munoz.pdf

Moreno J.L. (1993). Psicodrama. Buenos Aires: Lumen.

Nivoloni, J. M. (2008). El acompañamiento Terapéutico y la Psicosis. Revista de Psicoanálisis, Psicoterapia y Salud Mental, 1 (5), 31-41. Recuperado de http://cordescorporacion.cl/wp-content/uploads/2020/07/ El-Acompa\%C3\%B1amientoterapeutico-y-la-Psicosis. pdf

Ortega Ibarra, I. H., Ortega Ibarra, E. y Hernández Jiménez, A. (2019). Investigación de acción participativa ¿Una alternativa de investigación o una estrategia de solución? Universita. Ciencia, 61-70. Recuperado de https://www.researchgate.net/ publication/330509038_LA_INVESTIGACION_DE_ ACCION_PARTICIPATIVA_UNA_ALTERNATIVA_DE_INVESTIGACION_O_UNA_ESTRATEGIA_DE_SOLUUCION

Paredes-Chi, A. y Castillo-Burguete, M.T. (2018). "Caminante no hay [un solo] camino, se hace camino al andar": Investigación Acción Participativa y sus repercusiones en la práctica. Rev. Colomb. Soc., 41(1), 31 - 50. https://doi.org/10.15446/rcs.v41n1.66616

Pérez Ramírez, B. (Coord.). (2017). La relevancia de la mirada y la palabra en las estrategias de intervención en trabajo social. México: UNAM, ENTS.

Pérez Ramírez, B. (2019). Personas con discapacidad y privadas de su libertad en la Ciudad de México. Un estadístico de siete centros penitenciarios. México DF:
UNAM, ENTS.

Pulice, G. y Rossi, G. (1994). Acompañamiento Terapéutico: Aproximaciones a su conceptualización, presentación de material clínico. Buenos Aires: Polemos.

Red Navarra de lucha contra la pobreza y la exclusión social (2016). El acompañamiento como método de intervención en los procesos de inclusión: Nuevas reflexiones. España: Red Navarra.

Rivera, M. (24 de noviembre del 2007). Acompañantes terapéuticos: cuándo y dónde solicitarlos. El Litoral. Recuperado de https://www.ellitoral.com/index. php/diarios/2007/11/24/metropolitanas/AREA-05. html

Rosato, A., Angelino, A., Almeida, M., Angelino, C., Kippen, E., Sánchez, C., Spadillero, A., Vallejos, I., Zuttión, B. y Priolo, M. (2009). El papel de la ideología de la normalidad en la producción de discapacidad. Ciencia, Docencia y Tecnología, XX (39), 87-105. Recuperado de https://www.redalyc.org/ pdf/145/14512426004.pdf

Rossi, G.P. (2007). Acompañamiento Terapéutico. Lo cotidiano, las redes y sus interlocutores. Buenos Aires: Polemos.

Saiz Galdós, J. y Chevéz Mandelstein, A. (2009). La intervención Socio-Comunitaria en Sujetos con Trastorno Mental Grave y Crónico: Modelos Teóricos y Consideraciones Prácticas. Psychosocial Intervention, 18 (1), 75-88. Recuperado de http:// scielo.isciii.es/scielo.php?script=sci_arttext\&pi$\mathrm{d}=\mathrm{S} 1132-05592009000100009$

Segato, R. (2007). El color de la cárcel en América Latina. Nueva Sociedad, 208 (marzo-abril), 142-161. Recuperado de https://nuso.org/media/articles/downloads/3423_1.pdf

Segui García, G. A. (2013). Acompañamiento Terapéutico: Historia, Teoría y Clínica (Tesis de Pregrado). Universidad de Chile. Recuperado de http://repositorio. uchile.cl/handle/2250/130319

Serra Hurtado, J. L. (2003). Servicio de Salud Mental: Hospital de día. Alicante. Recuperado de https://es.slideshare.net/jlserra/hospital-de-dia

Sotomayor Acosta, J.O. (1990). Crítica a la peligrosidad como fundamento y medida de la reacción penal frente al inimputable. Nuevo Foro Penal, 12 (48), 1992013. Recuperado de https://publicaciones.eafit. edu.co/index.php/nuevo-foro-penal/issue/view/459

Tello Peón, N. (2010). Apuntes de Trabajo Social: trabajo social, disciplina del conocimiento. Recuperado de https://es.scribd.com/doc/75905790/Apuntes-Sobre-Intervencion-Social-Nelia-Tello-1

Urdapilleta Carrasco, J. y Limón Aguirre, F. (2018). Hacia una experiencia profunda dentro de la Investigación Acción Participativa. Revista colombiana de sociología, 41 (1), 111-131. Recuperado de https://dialnet.unirioja.es $/$ servlet $/$ articulo?codigo $=6583110$

Vásquez Aguilera, J. (2007). Inimputabilidad: análisis sobre la imposición judicial de las medidas de seguridad. Revista del Instituto de la Judicatura Federal, 23 (18), 287-301. Recuperado de https://www.ijf.cjf. gob.mx/publicaciones/revista/23/r23_18.pdf 